\title{
SAÚDE MENTAL DE ESTUDANTES DOS CURSOS DE GRADUAÇÃO EM ENGENHARIA
}

\author{
THE MENTAL HEALTH OF ENGINEERING STUDENTS \\ João Ernesto Moura Sobreira Bezerra ${ }^{1}$, Carlos Estêvão Rolim Fernandes², Layza Castelo Branco Mendes ${ }^{3}$, \\ Maria Salete Bessa Jorge ${ }^{4}$, Mayara Rocha Coelho ${ }^{5}$
}

DOI: 10.37702/REE2236-0158.v40p321-333.2021

\begin{abstract}
RESUMO
Parte-se do pressuposto que na atualidade o ambiente universitário pode ser disparador de situações estressoras. Além disso, em sua maioria, os estudantes universitários são adolescentes ou adultos jovens em processo de construção de sua identidade. Nesse momento, podem-se apresentar fragilidades psíquicas que podem desencadear prejuízos em seu aprendizado e na motivação em manter-se em seu curso. Alunos das áreas de tecnologias, por se tratar de uma área do conhecimento que se distancia das ciências da saúde, têm menos acesso a informações acerca dos aspectos relacionados à saúde psicossocial, tais como sintomas de adoecimento e formas de tratamento, entre outras. Diante desse cenário, o objetivo da pesquisa é analisar o estado atual da saúde mental de estudantes das áreas de engenharia. Trata-se de um estudo quantitativo. O campo empírico foi constituído por onze cursos de graduação em Engenharia que compõem o Centro de Tecnologia da Universidade Federal do Ceará (CT-UFC), e os sujeitos foram os estudantes do primeiro semestre. Os instrumentos de coleta de dados foram o questionário sociodemográfico, o Patient Health Questionnaire (PHQ-9) e o questionário de Qualidade de Vida da Organização Mundial da Saúde (WHOQoL-Bref), os quais foram aplicados a 418 estudantes. A relevância da pesquisa está relacionada à obtenção de informações consistentes sobre o estado atual da saúde mental dos estudantes do primeiro semestre do CT-UFC, tendo ficado explícito que muitos desses estudantes já entram no ensino superior com a qualidade de vida comprometida e com sintomas de depressão. Sugere-se ações da universidade para a promoção da boa qualidade de vida e promoção da saúde psíquica discente visando processos de aprendizagem eficazes.
\end{abstract}

Palavras-chave: saúde mental; engenharia; estudantes universitários.

\begin{abstract}
It is assumed that the university environment can be a trigger for stressful situations. In addition, most college students are teenagers or young adults in the process of building their identity. In this period of life, there may be psychical fragilities that can unleash their learning and the motivation to keep following his/her undergraduate program. Compared to Health Sciences students, those on Technology areas of knowledge have less access to information about psychosocial health, such as symptoms of illness and forms of treatment among others. Given this scenario, our goal is to investigate the current mental health state of engineering students. This is a quantitative case study research. The empirical field was the eleven engineering undergraduate programs of the Technology Center of the Federal University of Ceará (CT-UFC) and the subjects were first year students. The

\footnotetext{
${ }^{1}$ Médico Psiquiatra, Mestre em Saúde Coletiva, Universidade Estadual do Ceará, Campus Itaperi; joaoernesto@gmail.com

${ }^{2}$ Professor Associado, Doutor, Departamento de Integração Acadêmica e Tecnológica, Centro de Tecnologia, Universidade Federal do Ceará, Campus do Pici; estevao@ufc.br

${ }^{3}$ Professora Adjunta, Psicóloga, Doutora em Saúde Coletiva, Universidade Estadual do Ceará, Campus Itaperi; layza.mendes@uece.br

${ }^{4}$ Enfermeira, Professora e Pesquisadora bolsista produtividade CNPq 1B. Doutora em Enfermagem e Pós-Doutora em Saúde Coletiva, Universidade Estadual do Ceará, Campus Itaperi; maria.jorge@uece.br

5 Assistente Social, Graduanda e Mestranda em Psicologia, Bolsista IC/FUNCAP, Universidade Estadual do Ceará, Campus Itaperi; mayara.rocha@aluno.uece.br
} 
instruments and data collection technique were sociodemographic questionnaires, the Patient Health Questionnaire (PHQ-9) and the World Health Organization (WHOQoL-Bref) Quality of Life questionnaire applied to 418 students. The relevance of the research was to obtain consistent information about the current mental health state of the first year engineering students at the CTUFC, and it was explicit that many of these students are already entering higher education with symptoms of illness that provoke university actions for promotion of mental health in order to favor the learning process and the student's quality of life.

Keywords: mental health; engineering; university students.

\section{INTRODUÇÃO}

Há várias pesquisas sobre estudantes universitários, tanto no Brasil quanto em outros países. Entre estas, muitas focam em questões relacionadas à saúde mental (ANDRADE et al., 2016) e outras dão ênfase a aspectos gerais da qualidade de vida (SOUSA; JOSÉ; BARBOSA, 2013). Porém, a maioria dessas pesquisas têm sido realizadas com estudantes de áreas da saúde, como acadêmicos de Medicina, Enfermagem, Psicologia, entre outros. Imagina-se que a concentração de estudos dessa natureza na área da saúde devese ao fato de serem os pesquisadores oriundos dessas áreas, e, portanto, atentos a tais questões.

Poucos são os estudos como o de Castro (2017), que se deteve a analisar sintomas que comprometem a saúde mental de estudantes de engenharia. Acredita-se que isso se deve ao fato de haver pouca aproximação, na formação em engenharia, com as áreas da saúde (principalmente da saúde mental), estando alunos e professores distantes desse tema, percebendo-o apenas quando vivenciado por meio do próprio adoecimento. $\mathrm{O}$ estudo citado e os demais que verificaram estudantes das áreas da saúde apresentam conclusões semelhantes, quais sejam: não há apenas um fator isolado que desencadeia adoecimento mental e tais adoecimentos prejudicam a aprendizagem. O referido autor, na mesma linha de raciocínio de outros, conclui ser necessário que as universidades reconheçam a existência desses adoecimentos e que realizem atividades de cuidado. Além disso, tais pesquisas apontam a demanda por mais estudos nessa linha.
A vida universitária se inicia, geralmente, na fase final da adolescência ou no início da vida adulta. De acordo com o Inep (2017), o perfil etário de ingressantes é de 18 anos para os cursos de bacharelado e 21 anos para os cursos de licenciaturas. Trata-se de um período marcado por experiências individuais e coletivas que demandam mudanças de atitudes no sentido de mais responsabilização consigo e com a sociedade.

As experiências do referido período são importantíssimas para a construção desses sujeitos, dos quais se espera que, por meio do exercício de suas profissões, transformem realidades em nosso país. Entretanto, o mundo contemporâneo confronta os estudantes com exigências exacerbadas acerca da qualificação profissional. Por vezes, cobranças para desempenhos excepcionais são feitas quando os jovens ainda estão no início da vida universitária, em momento cujas experiências vividas influenciam a relação deles com o mundo. No Brasil, esse cenário fica acentuado devido ao fato de as melhores oportunidades de trabalho serem para pessoas com graduação de nível superior. Esse cenário descrito favorece processos de adoecimentos mentais.

Assim, vagas em universidades públicas e, principalmente, em cursos que socialmente são reconhecidos como sendo de boa empregabilidade e que dão status aos jovens como Medicina, Direito e Engenharias - são muito disputadas. Ao entrar na universidade, essas exigências não diminuem, pois, de acordo com a cultura das grandes universidades, para enfrentar o mercado de trabalho concorrido, é necessário se diferenciar desde o início do período de formação. Esse cenário faz parte do 
contexto sociocultural da atualidade no qual se vivencia fenômenos que afetam a formação profissional dos indivíduos. Entre elas, citamse incertezas quanto ao futuro profissional, competitividade, o desejo de acessar grande número de conhecimentos propagados continuamente e exigências que parecem almejar perfeições técnicas. Diante disso, os estudantes, além de cursarem disciplinas, buscam estágios, entram em laboratórios de pesquisa, tornam-se extensionistas, participam de eventos científicos e fazem cursos extracurriculares. Por vezes, essa rotina chega a ser exaustiva levando a adoecimentos psíquicos.

Diante disso, concorda-se com Moro, Valle e Lima (2005), que, nas universidades em que estudantes apresentam adoecimento psíquico no decorrer de sua trajetória acadêmica, deveria sempre haver programa de apoio psicológico que desse suporte. Tal serviço possibilitaria diminuir a prevalência e a incidência de sintomatologias depressivas e ansiosas, bem como ideações e tentativas de suicídio.

Justifica-se a necessidade de compreender e cuidar da saúde psicossocial de universitários por razão do contexto sociocultural em que esses sujeitos vivem. Ressalta-se que em grandes metrópoles a situação pode se agravar, pois demanda que esses sujeitos se submetam a situações de ter que diariamente percorrer grandes distâncias de casa ao trabalho e à universidade, diminuir o convívio com familiares, suprimir tempo de lazer e cuidados físicos, entre outras condições que levam à menor capacidade de manutenção da saúde psicossocial. Além disso, as incertezas relativas ao mundo acadêmico cada vez mais competitivo e individualista têm gerado sofrimento psíquico (FLACH, 2009).

Sobre os atores que compõem o corpo discente das universidades, destaca-se que, em sua maioria, são adolescentes ou adultos jovens ainda em processo de construção de sua identidade (BERTOL; SOUZA, 2010). Logo, possuem uma vulnerabilidade psíquica própria desse momento do desenvolvimento humano, que, ao ser somada às cobranças relativas à vida estudantil de nível superior, que exige maior autonomia e responsabilidade, pode desencadear sofrimentos geradores de adoecimentos psíquicos. Lembra-se com Lobato (2004) que os adolescentes e adultos jovens estão inseridos no contexto atual composto de cenários provisórios no mundo do trabalho, suscitando-lhes ansiedade excessiva.

Desse modo, o espaço universitário é uma espécie de limbo: é, por excelência, um lugar entre o conforto psíquico anteriormente garantido pelo papel de estudante de ensino médio - do qual a sociedade não exige que produza dinheiro - e o desconforto psíquico da obrigatoriedade ter de ser um produtor de ações socialmente úteis. Como lembra Foucault (2006), o trabalho é um dos fatores que tira os sujeitos do lugar no qual aquele que não é "normal" é colocado. Estar nesse estado de indefinição de um futuro profissional, a depender da interação com outros aspectos da vida desses sujeitos, pode gerar adoecimentos psíquicos.

Pergunta-se o que as universidades estão fazendo. Algumas já possuem programas de acompanhamento de alunos que apresentam sofrimento psíquico, tal como o Observatório Estudantil criado na Universidade Federal da Bahia (SAMPAIO, 2011). Outras possuem serviços não direcionados aos alunos, mas que os acolhe de forma incipiente, tal como na Universidade Federal do Mato Grosso (ASSIS; OLIVEIRA, 2010). Contudo, a prática mais comum tem sido ações isoladas apenas quando os estudantes já se encontram em processo de intenso sofrimento, quando não há mais como professores e colegas não perceberem a situação. Porém, na maioria das vezes, nem mesmo as atitudes isoladas existem, muito menos programas de cuidados com a saúde mental, com a prevenção de adoecimento ou com a promoção da saúde integral, que inclui a psíquica.

Cuidar da saúde mental não é apenas cuidar do adoecimento psicopatológico, pois a dicotomia saúde-doença hoje é superada pela ideia de saúde-doença-cuidado como um processo e, também, pela ideia de cuidado integral (ALMEIDA FILHO, 2008). Portanto, ao reconhecerem a necessidade de cuidar da saúde mental de estudantes, antes do início de adoecimentos, as universidades poderão evitar adoecimentos que interferem nas funções acadêmicas. Identificar alguém com 
comprometimento na saúde mental, antes de um prejuízo psicossocial e clínico maior, é premente para sua recuperação mais rápida e consistente.

Acredita-se que a universidade melhora sua produção científica e de formação de profissionais quando seus alunos, professores e servidores estão bem psiquicamente. Uma vez que o aluno esteja com a saúde psicossocial no nível máximo de sua capacidade, este terá maior êxito em suas habilidades de estudo e em sua formação, consequentemente.

Diante desses dados, no que tange à saúde mental dos estudantes de engenharia, verificouse a necessidade de ampliar os estudos sobre os adoecimentos psíquicos e a qualidade de vida deles. São escassos na comunidade científica tais dados. Há mais pesquisas sobre a saúde mental de estudantes das áreas da saúde. Considera-se que os estudantes da saúde estão imersos em cenários que têm como objeto de estudos os indivíduos e, por essa razão, podem ter mais habilidade para identificação e compreensão de sintomas de adoecimento. Já os estudantes de engenharias, que em seus cotidianos vivenciam com pouca frequência conteúdos relacionados ao humano e à saúde, ficam à mercê do olhar externo que identifique problemáticas. Contudo, um impasse fica estabelecido, pois seus próprios professores são, em sua maioria, também desprovidos de habilidades para lidar com tais questões.

Dessa forma, pergunta-se: qual o estado atual da saúde psíquica dos estudantes de engenharia? Os agravos em saúde mental de estudantes de engenharia se iniciam na universidade ou antes da entrada na universidade ou, ainda, iniciam-se antes da entrada na universidade e podem ser potencializados por vivências estressantes na academia? Poder responder a essas questões possibilitará a prevenção de adoecimentos psíquicos (FACUNDES; LUDERMIR, 2005; FUREGATO et al., 2006) e consequências, como reprovações por falta de motivação ou desânimo e dificuldades relacionais.

\section{ESTUDANTES DE ENGENHARIA E SAÚDE MENTAL}

Para compreender o cenário de pesquisas acerca da saúde mental de estudantes de engenharia foi realizada uma revisão de literatura, por meio de trabalhos encontrados na Biblioteca Virtual em Saúde (Bireme), com a seleção de publicações a partir dos termos: "saúde mental", "engenharia" e "estudantes". Colocou-se o filtro texto completo e, em seguida, no assunto principal, limitou-se à engenharia e estudantes, pois apareceram outros assuntos principais, mas que se distanciavam do objeto desta pesquisa. Restaram vinte e seis artigos, entre os quais se verificou, por meio da leitura dos resumos, que apenas três (encontrados na base de dados Medline) contemplavam o interesse desta pesquisa. Os excluídos usavam a palavra engenharia associada à Medicina. Deve-se destacar que outros estudos foram encontrados acerca desse tema, porém encontrados em outras buscas, e que foram utilizados na análise dos dados.

Entre os selecionados, apenas um deles aborda questões de saúde e os demais abordam motivação e desempenho acadêmico. A escolha deve-se aos cenários e às correlações que se pode fazer com a saúde mental.

Um dos estudos encontrados foi o de Mccomb e Kirkpatrick (2016), cujo objetivo foi comparar motivações para aprender em estudantes de enfermagem e engenharia em uma universidade estadunidense. O referido estudo abordou as mudanças no cenário do ensino superior e mostrou que os cursos avaliados possuem abordagens pedagógicas diferentes. Resultados mostraram que para ambos a potencialização da complexidade cognitiva de estudantes é diretamente proporcional à motivação para a aprendizagem. Outro dado mostrado no estudo foi que as disciplinas que trazem um aprendizado prático para ser aplicado à realidade apresentam diferentes estratégias pedagógicas, contudo todas exigem complexidade cognitiva, que, por sua vez, estimula a motivação dos estudantes para o avanço no processo de aprendizado. Sabe-se que a motivação está diretamente associada à saúde mental. 
A pesquisa de Kötter et al. (2014) tratou da promoção da saúde para estudantes universitários dos cursos de graduação em Medicina, Ciências, Tecnologia, Matemática e Engenharia em uma universidade na Alemanha. Tal pesquisa é um estudo longitudinal que, por meio de instrumentos psicométricos, avaliou os alunos anualmente. Autores partem da afirmação de que os riscos negativos sobre a saúde (física e mental) dos estudantes de Medicina são maiores que dos demais estudantes universitários. Acredita-se que essa afirmação deve ser confirmada com outras pesquisas, pois deve-se lembrar que eles têm mais acesso a informações sobre saúde, e, portanto, podem ter mais aptidão para autopercepção de sintomas.

$\mathrm{O}$ referido estudo, embora tenha sido comparativo, pareceu focar mais os estudantes de Medicina, sendo os demais apenas balizadores dos primeiros. $\mathrm{O}$ primeiro ano da pesquisa mostrou índices semelhantes de saúde geral para estudantes de Medicina e os demais, com referenciais positivos de $79,7 \%$ e $90,9 \%$, respectivamente. Já especificamente sobre a saúde mental, esses valores são positivos e ainda mais próximos, $86,3 \%$ para estudantes de Medicina e $88,3 \%$ para estudantes dos demais cursos citados. Thomas et. al. (2004) mostraram também que a satisfação com a vida e a atividade física parecem ter sido fator que preveniu a saúde geral dos estudantes de ambos os cursos que também foi verificada ao final da pesquisa.

O estudo de Freeman et al. (2014) foi realizado com estudantes de Ciências, Matemática e Engenharia de universidades estadunidenses e canadenses. A pesquisa comparou desempenho acadêmico, incluindo reprovação em disciplinas em que eram utilizadas abordagens tradicionais de ensino e em disciplinas que se pautavam em metodologias ativas de ensino. Os achados mostraram que o processo de aprendizado por meio de metodologias ativas aumenta o desempenho dos alunos, bem como metodologias tradicionais aumentam o índice de reprovação. Os pesquisadores concluíram que, havendo substituição de aulas tradicionais por metodologias ativas, haveria mais interesse dos alunos pelos cursos pesquisados
Os estudos acima citados mostram importantes dados que devem ser levados em consideração sobre a formação acadêmica de estudantes de engenharias, a correlação com a saúde mental e, consequentemente, com a formação. Reflexões são instigadas nesses estudos aqui citados, contudo, é preciso afirmar que ainda é pobre a literatura que trate de problemáticas de saúde mental entre estudantes universitários de engenharia, mesmo internacionalmente, e a consequente correlação com a qualidade da formação do profissional.

\section{MÉTODO}

Inicialmente, esta pesquisa fez uso de abordagem quantitativa e qualitativa do tipo estudo de caso, exploratório e transversal, pois pretendeu analisar questões sobre o tema da saúde mental dos estudantes de engenharia. $\mathrm{O}$ campo empírico foi constituído por onze cursos de graduação de Engenharia que compõem o Centro de Tecnologia da Universidade Federal do Ceará (CT-UFC), localizado na capital, Fortaleza. Os sujeitos pesquisados foram 418 estudantes do primeiro semestre dos cursos de engenharia, que compuseram os dados quantitativos; e 12 alunos do quarto ao décimo segundo semestre do CT-UFC, que compuseram os dados qualitativos. Este estudo aborda o recorte dos dados quantitativos coletados com os alunos do primeiro semestre. A amostragem seguiu um procedimento probabilístico estratificado, sendo os estratos os próprios cursos de graduação em engenharia. Os formulários foram aplicados em disciplinas obrigatórias para ingressantes no curso. A aleatoriedade da amostra reside no fato de se tomar os alunos presentes em sala de aula num certo dia, sendo que não houve aviso prévio sobre a aplicação dos instrumentos. Todos os professores dessas disciplinas deram sua expressa anuência para a realização da coleta de dados. Nenhum aluno foi obrigado a responder aos questionários e todos os respondentes assinaram Termo de Consentimento Livre e Esclarecido (TCLE). 
Tabela 1 - Distribuição dos estudantes que responderam aos questionários por curso

Curso

Engenharia Ambiental

Engenharia Civil

Engenharia da Computação

Engenharia Elétrica

Engenharia Mecânica

Engenharia Metalúrgica

Engenharia de Petróleo

Engenharia de Produção Mecânica

Engenharia Química

Engenharia de Energias Renováveis

Engenharia de Telecomunicações

Total

Fonte: elaborada pelos autores.

A coleta dos dados quantitativos foi realizada por meio de questionário sociodemográfico elaborado pelos pesquisadores, do questionário de Qualidade de Vida da Organização Mundial de Saúde (OMS) - versão abreviada (WHOQoL-bref) - e do questionário Patient Health Questionnaire (PHQ-9). Esses instrumentos foram aplicados presencialmente.

O WHOQOL-bref, já validado no Brasil, é formado por 26 perguntas, cujas respostas seguem uma escala de 1 a 5 ; quanto maior a pontuação maior a qualidade de vida. A primeira questão indaga sobre como a pessoa avaliaria sua qualidade de vida e a segunda sobre o quão ela está satisfeita com a própria saúde, sendo ambas, portanto, mais abrangentes. As demais 24 perguntas formam quatro domínios: saúde física, psicológico, relações sociais e ambiente (FLECK, 2000; KLUTHCOVSKY; KLUTHCOVSKY, 2009) (Quadro 1).

Quadro 1 - Domínios e facetas correspondentes no WHOQOL-bref

\begin{tabular}{|c|c|}
\hline DOMÍNIO & FACETAS \\
\hline Saúde física & Dor física \\
& Capacidade de trabalho \\
& Tratamento médico \\
& Atividades diárias \\
& Energia e cansaço \\
& Mobilidade \\
& Sono e repouso \\
\hline Domínio & Sentimentos positivos \\
psicológico & Sentimentos negativos \\
& Crenças pessoais \\
& Autoestima \\
& Concentração e memória \\
\hline
\end{tabular}

\begin{tabular}{|c|c|}
\hline & Aceitação da aparência física \\
\hline $\begin{array}{c}\text { Domínio das } \\
\text { relações } \\
\text { sociais }\end{array}$ & Relações pessoais \\
Apoio social \\
Vida sexual \\
\hline $\begin{array}{c}\text { Domínio do } \\
\text { ambiente }\end{array}$ & Segurança física \\
& Ambiente físico \\
& Meios de transporte \\
& Acesso a serviços de saúde \\
& Recursos financeiros \\
& Condições de moradia \\
& Oportunidades de novas \\
& informações \\
\hline
\end{tabular}

Fonte: elaborado pelos autores.

Seguindo as transformações sugeridas em Whoqol Group (1995), os resultados são apresentados por domínio na escala de 0 a 100 , em direção positiva (quanto mais alto o escore, melhor a qualidade de vida).

Além de verificar a qualidade de vida dos sujeitos da pesquisa, pensou-se ser relevante verificar, por meio de um instrumento qualificado, a existência de sintomas relacionados a adoecimentos mentais. Foi por essa razão que se utilizou também o PHQ-9, que identifica sintomas depressivos, o qual foi validado no Brasil para triagem na população geral. Tal instrumento é formado por nove perguntas que constituem cada sintoma para o episódio depressivo maior, segundo o Manual Diagnóstico e Estatístico dos Transtornos Mentais IV (DSM-IV) (SANTOS et al., 2013).

A importância de questionários de rastreio dos quadros depressivos em amostras populacionais se deve ao fato de os transtornos depressivos serem condições bastante prevalentes na população geral e estão associados a graves prejuízos no funcionamento físico, social e funcional. Sujeitos que sofrem desses transtornos muitas vezes não procuram ajuda profissional e quando buscam relatam apenas sintomas somáticos, passando sua depressão muitas vezes despercebida (MARTIN et al., 2006).

Os dados foram tabulados em planilha eletrônica LibreOffice Calc, passaram por alguns tratamentos realizados com uso da plataforma aberta GNU-Octave e posteriormente foram analisados criticamente. 


\section{RESULTADOS E DISCUSSÃO}

Registrou-se na amostra uma prevalência de sujeitos do sexo masculino, dado esperado pelo fato de os cursos de engenharia serem mais procurados por homens. Contudo, no curso de Engenharia Ambiental, disciplina recente no Brasil se comparada com outras engenharias mais tradicionais, a quantidade de sujeitos do sexo feminino foi maior. Sobre a faixa de idade, a maioria dos estudantes, 76,32\% (319), está na faixa etária entre 18 e 21 anos, o que se explica por serem alunos do primeiro semestre dos cursos. Acredita-se que é devido a essa pouca idade que a maioria deles também respondeu morar com pais ou responsáveis.

A percentagem semelhante entre alunos que estudaram em escolas públicas e privadas é explicada pelo fato de o acesso à UFC por vestibular seguir o critério de cotas em $50 \%$ para alunos de escolas públicas e $50 \%$ para alunos de escolas privadas (UNIVERSIDADE FEDERAL DO CEARÁ, 2018).

Tabela 2 - Resultados dos dados sociodemográficos obtidos de questionário respondido pelos estudantes das engenharias do CT-UFC do primeiro semestre

\begin{tabular}{lr}
\hline Variável & Número de alunos $(\%)$ \\
\hline Sexo & $323(77,3)$ \\
Masculino & $95(22,7)$ \\
Feminino & \\
Idade & $65(15,55)$ \\
$<18$ & $319(76,32)$ \\
$18-21$ & $26(6,22)$ \\
$22-25$ & $8(1,91)$ \\
$>25$ & \\
Naturalidade & $276(66,03)$ \\
Capital & $102(24,4)$ \\
Interior & $34(8,13)$ \\
Outros estados & $5(1,2)$ \\
Outros países & $1(0,24)$ \\
Não respondeu & \\
Com quem mora & $144(34,45)$ \\
Família [pais+irmão(s)] & $94(22,49)$ \\
Pais & $67(16,03)$ \\
Mãe & $6(1,43)$ \\
Pai & $5(1,2)$ \\
Avós & $10(2,39)$ \\
Irmãos & $3(0,72)$ \\
Cônjuge & $41(9,81)$ \\
Outros parentes & $26(6,22)$ \\
Amigos & $15(3,59)$ \\
Sozinho & $7(1,67)$ \\
Não responderam & \\
Cursou o ensino médio em escola &
\end{tabular}

\begin{tabular}{lr} 
Pública & $196(46,89)$ \\
Privada & $222(53,11)$ \\
Já cursou outra faculdade & \\
Sim & $79(18,9)$ \\
Não & $339(81,1)$ \\
Trabalha & \\
Sim & $25(5,98)$ \\
Não & $393(94,02)$ \\
Total de alunos & $\mathbf{4 1 8}(\mathbf{1 0 0})$ \\
\hline
\end{tabular}

Fonte: elaborada pelos autores.

Sobre a análise do questionário WHOQOL-bref, o resultado médio ficou em 58,9 , resultado baixo se comparado com algumas médias referidas em outros estudos com estudantes universitários que utilizaram o mesmo instrumento. Teixeira (2008), avaliando estudantes de diversos cursos de graduação superior, incluindo Engenharia Civil e de Produção Mecânica, verificou que eles apresentavam média de qualidade de vida global pelo WHOQOL-bref de 65,08. Chazan e Campos (2013) mostraram média de 66 com o mesmo instrumento para a qualidade de vida global entre 394 estudantes de Medicina. Chehuen Neto et al. (2008) estudaram 232 estudantes de Medicina e Direito e a análise mostrou, com o mesmo questionário, que $90 \%$ daqueles de Direito avaliaram sua qualidade de vida como "boa" ou "muito boa". Em outro estudo, com 136 estudantes de Psicologia, a qualidade de vida global média ficou em 74,9, também com o WHOQOL-bref (COSTA et al., 2008). Já entre 140 alunos do curso de Administração, com o mesmo instrumento, a média ficou em 56,86 entre aqueles que trabalhavam e estudavam, e em 60,46 entre os que não trabalhavam (TOMBOLATO, 2005).

A seguir, nos Gráficos 1 a 5, apresentamse as medianas dos resultados em valores brutos (sem transformações) dos diferentes domínios analisados pelo WHOQOL-bref. Por essa razão, os indicadores variam de 1 a 5 . Quanto ao domínio saúde física, conforme o Gráfico 1 a seguir, os quesitos em que aparecem as maiores dificuldades são energia e cansaço, sono e repouso, capacidade de trabalho e atividades diárias. O Gráfico 2, relativo ao domínio psicológico, mostra que os estudantes respondentes consideram como de pior qualidade: concentração, memória, autoestima e sentimentos negativos (mau humor, 
desespero, ansiedade e depressão) e positivos (segundo a pergunta sobre o quanto se aproveita a vida).

O Gráfico 3, que aborda o domínio de relações sociais, mostra que as relações pessoais e o apoio social impactam mais positivamente na qualidade de vida dos sujeitos respondentes. Além desses dois pontos avaliados, o terceiro, que é sobre vida sexual, também mostrou bons resultados. O Gráfico 4 mostra que, entre os oito quesitos do domínio ambiente, apenas as condições de moradia e a oportunidade de novas informações traziam mais qualidade de vida; os demais quesitos mostram neutralidade na avaliação pautada na percepção que os estudantes têm acerca de sua qualidade de vida.

Gráfico 1 - Mediana das avaliações dos entrevistados nos quesitos do domínio saúde física do WHOQOL-bref entre todos os estudantes de

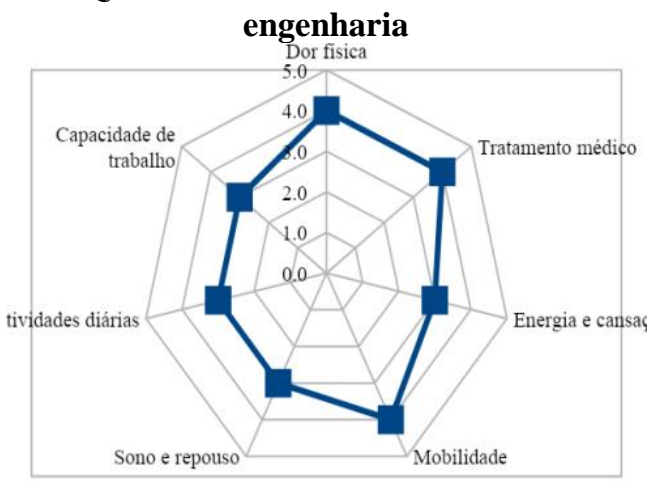

Fonte: elaborado pelos autores.

Gráfico 2 - Mediana das avaliações dos entrevistados nos quesitos do domínio psicológico do WHOQOL-bref entre todos os estudantes de

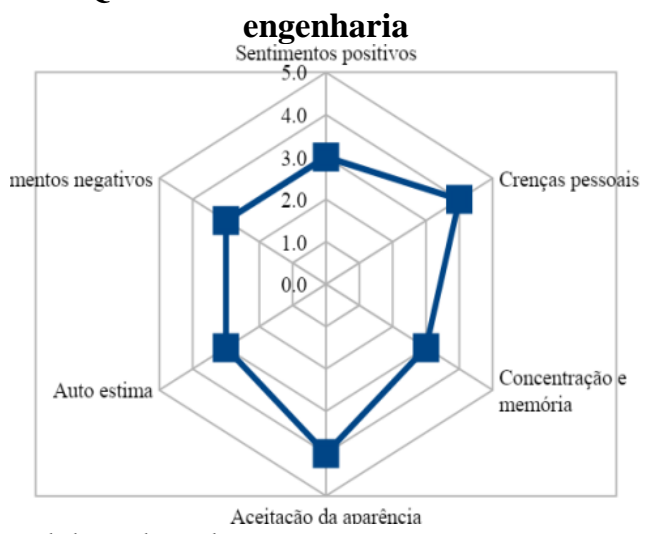

Fonte: elaborado pelos autores.
Gráfico 3 - Mediana das avaliações dos entrevistados nos quesitos do domínio relações sociais do WHOQOL-bref entre todos os estudantes

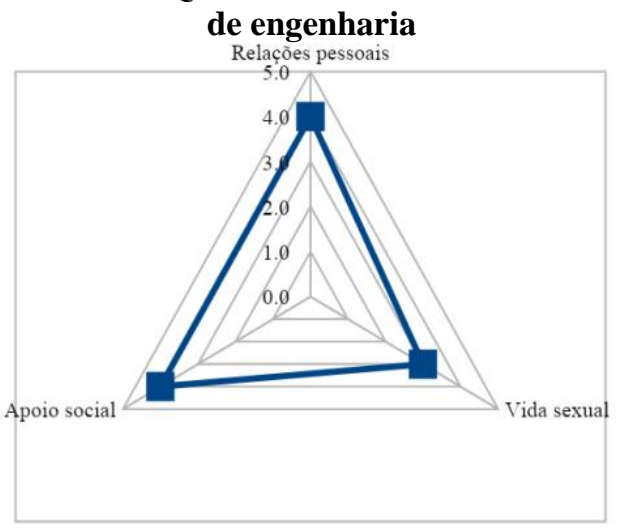

Fonte: elaborado pelos autores.

Gráfico 4 - Mediana das avaliações dos entrevistados nos quesitos do domínio ambiente do WHOQOL-bref entre todos os estudantes de

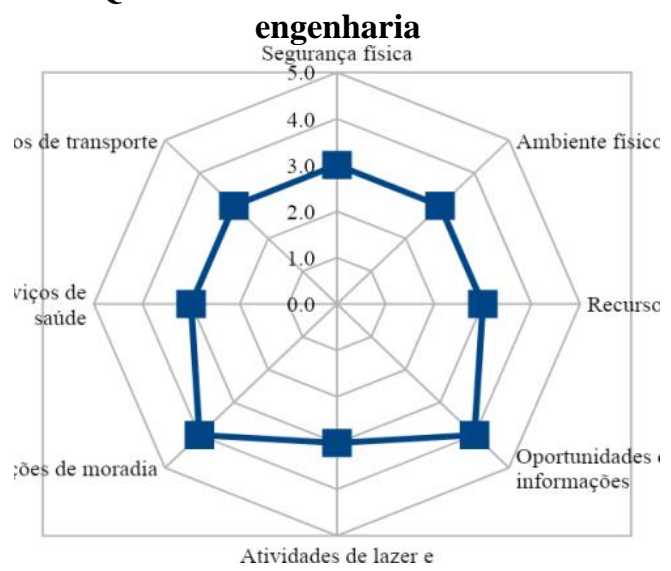

Fonte: elaborado pelos autores.

Sobre os resultados do questionário PHQ9 respondido pelos estudantes de engenharia, estimou-se uma possível prevalência de depressão naquela população - que deve ser confirmada por meio de consulta médica. Tal hipótese se fundamenta principalmente nos dados acerca do grau de dificuldade na realização de tarefas, de tomar conta das coisas de casa e de se relacionar com as pessoas.

O Gráfico 5 mostra 18,6\% e 39,5\% dos alunos com, respectivamente, nenhum ou mínimos sintomas depressivos e sintomas suaves, correspondendo àqueles, portanto, a sem depressão provável. Considera-se, assim, que os sintomas classificados como moderados, moderadamente grave e grave são aqueles que denotam possibilidade maior de o indivíduo estar com depressão clinicamente relevante. Considerando o grupo de todos os estudantes do primeiro semestre das engenharias do CT-UFC, 
41,9\% deles apresentam essas gravidades de sintomas depressivos.

Gráfico 5 - Percentagem dos estudantes de engenharia e a gravidade demonstrada nos sintomas de depressão segundo respostas ao questionário PHQ-9
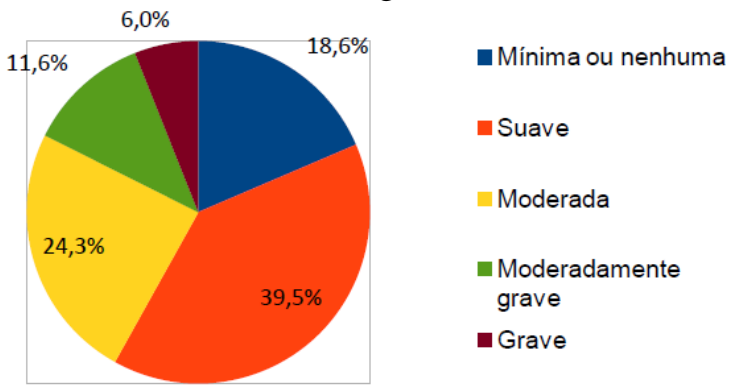

Fonte: elaborado pelos autores.

O Gráfico 6 mostra que a grande maioria $(73,5 \%)$ dos estudantes das engenharias do CTUFC apresenta grau baixo ou muito baixo de dificuldade para a realização do seu trabalho, dar conta das tarefas de casa e relacionar-se com os outros. Preocupa, contudo, que 26,5\% desses estudantes tenham graus médio ou alto de dificuldade, denotando prejuízo psicossocial para essa população.

O sujeito com depressão clínica, ou seja, aquela que requer cuidados de profissionais de saúde - tais como psiquiatras, psicólogos, terapeutas ocupacionais, entre outros -, apresenta sintomas associados a prejuízo psicossocial significativo (AMERICAN PSYCHIATRIC ASSOCIATION, 2014). Logo, pode-se entender que pelo menos $26,5 \%$ mostraram sintomas significativos relativos a transtorno depressivo maior e, portanto, deveriam ser avaliados por profissionais da área de saúde mental para se poder confirmar ou refutar tal diagnóstico.
Gráfico 6 - Dificuldade dos estudantes de engenharia para a realização do trabalho, lidar com as tarefas domésticas e relacionar-se com as pessoas, segundo respostas ao questionário PHQ-9, em percentagem

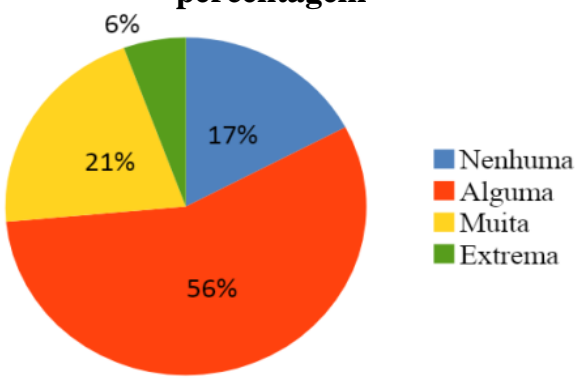

Fonte: elaborado pelos autores.

\section{CONCLUSÕES}

Os dados colhidos do questionário WHOQOL-bref qualificaram o grau de satisfação com a vida dos estudantes do primeiro semestre de graduação das faculdades de engenharia da UFC, com dados preocupantes e que merecem mais atenção e investigação por parte da instituição. Ainda mais preocupantes são os dados do questionário PHQ-9, que mostraram que alguns desses estudantes apresentaram sintomas depressivos e, portanto, precisam ser cuidados com celeridade. A universidade, como instituição acolhedora desses alunos, deve, em parte, preocupar-se com eles e ajudá-los na busca de ajuda especializada. Tal cenário constatado pode se agravar com o decorrer dos anos e com o envolvimento em mais atividades acadêmicas.

Lembra-se que os estudantes que responderam aos questionários são do primeiro semestre. Por conseguinte, parece ter havido pouca influência das atividades acadêmicas para o estado de sofrimento ou baixa qualidade em vários aspectos da vida dos sujeitos. Isso pode denotar, outrossim, que tais estudantes que cursam engenharias já podem ter chegado à universidade com sintomas depressivos e comprometimentos na qualidade de vida. Cabe, portanto, discutir, por exemplo, o processo de entrada na universidade como uma possível causa ou fator motivador para o estresse dos estudantes ainda no ensino médio.

Assim, pergunta-se: será que a preparação dos vestibulandos para as provas de acesso ao 
ensino superior está gerando sofrimento psíquico e comprometimentos na qualidade de vida, que, consequentemente, podem gerar processos de adoecimentos?

Logo, é premente que as coordenações de graduações e a direção de departamento, que são corresponsáveis por esse cenário, preocupem-se com a saúde mental de seus alunos desde sua entrada na universidade, promovendo ações preventivas. Inclui-se, entre essas ações, a organização de projetos pedagógicos de curso, de forma a não levar os estudantes a excessos de atividades que não contribuam para o processo de ensino e aprendizagem. Ademais, os métodos de avaliação devem estar em concordância com diversas mudanças no ensino superior no Brasil e no restante do mundo. A tendência tem sido se pautar em metodologias ativas, que são comprovadamente mais eficazes. Tais ações podem compensar as pressões sofridas no ensino médio no Brasil, em que pouco se preocupa com as subjetividades, focando apenas nos resultados de aprovações. Logo, os currículos dos cursos de Engenharia de Instituições de Ensino Superior podem ser pensados de forma a garantir momentos de descanso para o aluno e aulas em horários que respeitem as necessidades fisiológicas do ser humano, como tempos de sono e alimentação suficientes.

Além disso, sugere-se o estímulo à formação de núcleos de acompanhamento pedagógico em todas os cursos de graduação que tiveram sujeitos participantes desta pesquisa. A finalidade dessa proposta é de acompanhar o desenvolvimento da aprendizagem ao longo do curso, incluindo o possível surgimento de sofrimentos relacionados com a universidade ou que atrapalhem os ganhos acadêmicos de cada um.

Para pessoas que manifestem comprometimentos na qualidade de vida e/ou sintomas depressivos, deve haver meios de acolhimento de profissionais de saúde capacitados e vinculados à UFC. É preciso também que os estudantes saibam que passos podem seguir para isso.

Cabe também às direções e coordenações de graduações capacitar os professores a identificarem alunos cujo comprometimento na qualidade de vida ou a presença de sintomas de depressão expressem-se no ambiente universitário, prejudicando sua trajetória acadêmica. Sabe-se que professores, coordenadores ou diretores não podem diagnosticar doenças, mas podem ter a empatia de perceber um mal-estar e sugerir a busca de apoio. Ademais, direções devem promover educação continuada em recursos pedagógicos, como o uso cada vez maior de novas metodologias de ensino-aprendizagem, como as chamadas metodologias ativas. Mas o corpo docente também deve se corresponsabilizar em buscar esse conhecimento, cobrando das IES o espaço para que essa problemática seja remediada.

\section{REFERÊNCIAS}

ALMEIDA FILHO, N. A universidade nova no século XXI. In: ALMEIDA FILHO, N; SANTOS B. S. A universidade no século XXI: para uma universidade nova. Coimbra: Almedina, 2008. p. 79-184.

AMERICAN PSYCHIATRIC ASSOCIATION. Manual diagnóstico e estatístico de transtornos mentais: DSM-5 - 5. ed. Porto Alegre: Artmed, 2014.

ANDRADE, A. do S. et al. Vivências acadêmicas e sofrimento psíquico de estudantes de psicologia. Psicol. ciênc. prof., v. 36, n. 4, p. 831-846, 2016.

ASSIS, A. D. de; OLIVEIRA, A. G. B. de. Vida universitária e saúde mental: atendimento às demandas de saúde e saúde mental de estudantes de uma universidade brasileira. Cad. bras. saúde ment., v. 2, n.4-5, p.159177, 2010.

BERTOL, C. E.; SOUZA, M. de. Transgressões e adolescência: individualismo, autonomia e representações identitárias. Psicol. ciênc. prof., v. 30, n. 4, p. 824-839, 2010.

CABRAL, C. G. As mulheres nas escolas de engenharia brasileiras: história, educação e futuro. Cad. gên. tecnol., v. 1, n. 4, p. 9-19, 2005. 
CASTRO, V. R. Reflexões sobre a saúde mental do estudante universitário: estudo empírico com estudantes de uma instituição pública de ensino superior. Revista Gestão em Foco, n. 9, p. 380-481, 2017.

CHAZAN, A. C. S.; CAMPOS, M. R. Qualidade de vida de estudantes de medicina medida pelo WHOQOL-bref - UERJ, 2010. Rev. bras. educ. med., v. 37, n. 3, p. 376-384, 2013.

CHEHUEN NETO, J. A. et al. Qualidade de vida dos estudantes de medicina e direito. HU Rev., v. 34, n. 3, p. 197-203, 2008.

COSTA, C. C. da et al. Qualidade de vida e bemestar espiritual em universitários de psicologia. Psicol. est., v. 13, n. 2, p. 249-255, 2008.

CRISTÓVÃO, F. C. C. Sofrimento emocional, stress e depressão em estudantes universitários. 2012. 50f. Dissertação (Mestrado em Psicologia Clínica) Departamento de Educação da Universidade de Aveiro, Aveiro (Portugal), 2012.

FACUNDES, V. L. D.; LUDERMIR, A. B. Transtornos mentais comuns em estudantes da área de saúde. Rev. bras. psiquiatr., v. 27, n. 3, p. 194-200, 2005.

FLACH, L. Sofrimento psíquico no trabalho contemporâneo: analisando uma revista de negócios. Psicol. soc., v. 21, n. 2, p. 193-202, 2009.

FLECK, M. P. A. et al. Aplicação da versão em português do instrumento abreviado de avaliação da qualidade de vida "WHOQOLbref”. Rev. Saúde Pública, v. 34, n. 2, p. 178$83,2000$.

FOUCAULT, M. Loucura e Sociedade. In: Ditos e Escritos: Problematização do sujeito: Psicologia, Psiquiatria e Psicanálise, vol I. Rio de Janeiro: Forense Universitária, 2006.

FREEMAN, S., et al. Active learning increases student performance in science, engineering, and mathematics. Proc. natl. acad. sci., v. 111, n. 23, 2014.

FUREGATO, A. R. F., et al. Depressão e autoestima entre os acadêmicos de Enfermagem.
Rev. psiquiatr. clín., v. 33, n. 5, p. 239-44, 2006.

INEP. INSTITUTO NACIONAL DE ESTUDOS E PESQUISAS EDUCACIONAIS ANÍSIO TEIXEIRA. Censo da educação superior: notas estatísticas 2017. Brasília, 2017.

KLUTHCOVSKY, A. C. G. C.; KLUTHCOVSKY, F. A. O WHOQOL-bref, um instrumento para avaliar qualidade de vida: uma revisão sistemática. Rev. psiquiatr. Rio Gd. Sul, v. 31, n. 3 (supl.0), 2009.

KÖTTER, T. et al. Health-promoting factors in medical students and students of science, technology, engineering, and mathematics: design and baseline results of a comparative longitudinal study. BMC med. educ., v.14, p. 134, 2014.

LOBATO, C. R. P. S. O significado do trabalho para o adulto jovem no mundo do provisório. Rev. psicol. UnC, v. 1, n. 2, p. 44-53, 2004.

MARTIN, A. et al. Validity of the Brief Patient Health Questionnaire Mood Scale (PHQ-9) in the general population. Gen. hosp. psychiat., v. 28, p. 71-77, 2006.

MCCOMB, S. A.; KIRKPATRICK, J. M. Impact of pedagogical approaches on cognitive complexity and motivation to learn: Comparing nursing and engineering undergraduate students. Nurs. outlook., v. 64, v. 1, p. $37-48,2016$.

MENDES, L. C. B. A interdisciplinaridade na educação do profissional da saúde no Brasil: estudo do caso "Bacharelado interdisciplinar em saúde" da Universidade Federal da Bahia. 2013. 469f. Tese (Doutorado em Saúde Coletiva) - Universidade Estadual do Ceará, Centro de Ciências da Saúde, Fortaleza. 2013.

MORO, A.; VALLE, J. B. do; LIMA, L. P. de. Sintomas depressivos nos estudantes de medicina da Universidade da região de Joinville(SC). Rev. bras. educ. méd., v 29, n $2,2005$.

SAMPAIO, S. M. R., org. Observatório da vida estudantil: primeiros estudos [online]. Salvador: EDUFBA, 2011. 
SANTOS, I. S. et al. Sensibilidade e especificidade do Patient Health Questionnaire-9 (PHQ-9) entre adultos da população geral. Cad. saúde pública, v. 29, n. 8, p. 1533-1543, 2013.

SOUSA, N. A. de; MARQUES, I. R. Período de estudos e qualidade de vida do estudante de enfermagem. Rev. bras. qual. vida, v. 2, n. 2, p. $1-8,2010$.

SOUSA, T. F. de; JOSÉ, H. P. M.; BARBOSA, A. R. Condutas negativas à saúde em estudantes universitários brasileiros. Ciênc. saúde coletiva, v. 18, n. 12, p. 3563-3575, 2013.

SOUZA, D. C. de. Condições emocionais de estudantes universitários: estresse, depressão, ansiedade, solidão e suporte social. 2017. 90f. Dissertação (Mestrado em Psicologia Clínica) - Universidade Federal do Triângulo Mineiro, Uberaba, 2017.

TEIXEIRA, A. M. F. Entre a escola pública e a universidade: longa travessia para jovens de origem popular. In: SAMPAIO, S. M. R. (Org.). Observatório da vida estudantil: primeiros estudos [online]. Salvador: EDUFBA, 2011, p. 27-51.

TEIXEIRA, R. C. Qualidade de vida e eficácia adaptativa em estudantes universitários.
2008. 91f. Dissertação (Mestrado em Psicologia da Saúde) - Universidade Metodista de São Paulo, São Bernardo do Campo, 2008.

TOMBOLATO, M. C. R. Qualidade de vida e sintomas psicopatológicos do estudante universitário trabalhador. 2005. 96f. Dissertação (Mestrado em Psicologia Clínica) - Pontifícia Universidade Católica de Campinas, Campinas, 2005.

UNIVERSIDADE FEDERAL DO CEARÁ. Próreitoria de Graduação. Coordenação do Processo Seletivo Sisu. Notas de Corte Processo Seletivo Sisu 1\%/2017. Disponível em: < http://www.sisu.ufc.br/notas-de-corte/>. Acesso em: 24 de set. 2018.

Secretaria de Tecnologia da Informação. Informações sobre cotas. Disponível em: <http://www.sisu.ufc.br>. Acesso em: $24 \mathrm{de}$ set. 2018.

WHOQOL GROUP. The World Health Organization quality of life assessment (WHOQOL): Position paper from the World Health Organization. Social science \& medicine., v. 41, n. 10, p. 1403-1409, 1995.

\section{DADOS BIOGRÁFICOS DOS AUTORES}

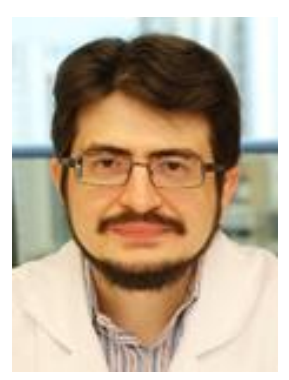

João Ernesto Moura Sobreira Bezerra - Médico pela Universidade Federal do Ceará (2006). Psiquiatra pela Escola de Saúde Pública do Ceará (2011). Psicogeriatra e Especialista em dependência química pela Escola Paulista de Medicina da Universidade Federal de São Paulo (2012). Mestre em Saúde Coletiva pela Universidade Estadual do Ceará (2018). Atua e pesquisa sobre a saúde mental dos estudantes universitários.

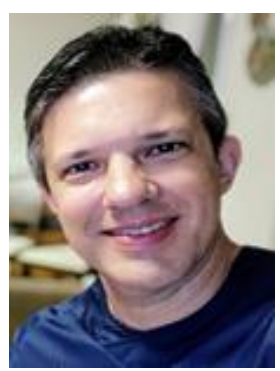

Carlos Estêvão Rolim Fernandes - Engenheiro Eletricista pela Universidade Federal do Ceará (1999). Mestre em Engenharia Elétrica pela UFC (2002) e Doutor pela Universidade de Nice Sophia Antipolis, França (2008), na área de Automação e Processamento de Sinais. Atualmente é professor associado da UFC nos cursos de Engenharia de Energias e Meio Ambiente, pelo Departamento de Integração Acadêmica e Tecnológica. Além das questões atuais da educação em Engenharia, seus interesses científicos incluem o processamento estatístico da informação, identificação de sistemas, análise de séries temporais e modelagem estocástica. 

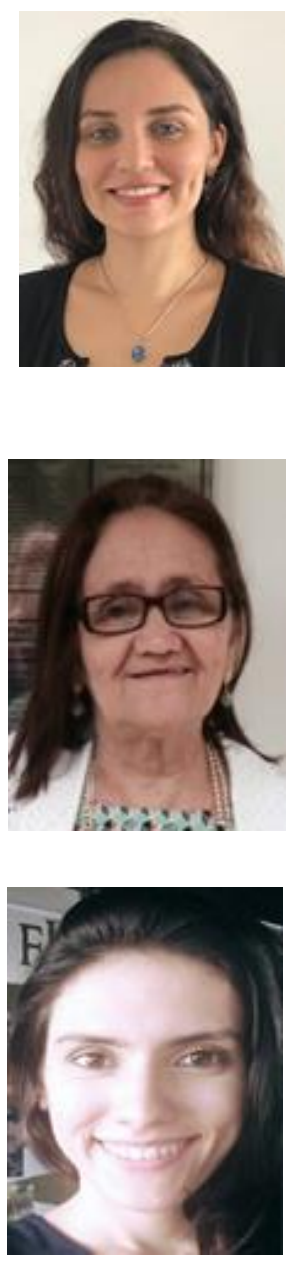

Layza Castelo Branco Mendes - Psicóloga pela Universidade Federal do Ceará (2003). Especialista em Psicologia da Adolescência pela Universidade de Fortaleza (2005). Especialista em Psicodrama Clínico pela Faculdade Integrada do Ceará (2007) e pela Federação Brasileira de Psicodrama (2007). Mestre em Psicologia pela Universidade de Fortaleza (2007). Doutora em Saúde Coletiva pela Universidade Estadual do Ceará (2013). Atualmente é professora adjunta da Universidade Estadual do Ceará e Coordenadora do Laboratório de Sociedades, Subjetividades e Humanismo (LASSU). Atua e pesquisa sobre a saúde mental dos estudantes universitários.

Maria Salete Bessa Jorge - Enfermeira, Doutora em Enfermagem pela Universidade de São Paulo, Pós-Doutorado em Saúde Coletiva pela Unicamp. Atualmente é professora titular da Universidade Estadual do Ceará, pesquisadora bolsista produtividade CNPq 1B. Líder do Grupo de Pesquisa em Saúde Mental, Família e Práticas de Saúde e Enfermagem do CNPq. Docente do Programa de PósGraduação em Saúde Coletiva - UECE. Coordenadora do Mestrado Profissional em Gestão em Saúde da Universidade Estadual do Ceará - MEPGES/UECE.

Mayara Rocha Coelho - Assistente Social pela Universidade Estadual do Ceará (2014). Graduanda em Psicologia pela Universidade Estadual do Ceará. Mestranda em Psicologia pela Universidade Federal do Ceará. Integrante do Laboratório de Sociedades, Subjetividades e Humanismo (LASSU), atuando como Bolsista IC/FUNCAP. Pesquisa sobre saúde mental dos estudantes universitários. 